\title{
COMPOSTO ORGÂNICO EM MANGUEIRAS (Mangifera indica L.) CULTIVADAS NO SEMIÁRIDO DO NORDESTE BRASILEIRO ${ }^{1}$
}

\author{
DAVI JOSÉ SILVA², MARIA APARECIDA DO CARMO MOUCO 3 , \\ CARLOS ALBERTO TUÃO GAVA ${ }^{4}$, VANDERLISE GIONGO ${ }^{5}$, JOSÉ MARIA PINTO ${ }^{6}$
}

RESUMO - O objetivo deste trabalho foi avaliar as características químicas do solo, a concentração de nutrientes nas folhas e a produção de mangueiras cultivadas em sistema orgânico. Três compostos denominados A, B e C foram aplicados em três doses cada $\left(0 ; 5\right.$ e $\left.10 \mathrm{t} \mathrm{ha}^{-1}\right)$, em área comercial de produção de mangueira em cultivo orgânico. O experimento foi conduzido no delineamento de blocos ao acaso, em fatorial 3 x 3 ( 3 compostos x 3 doses), com três repetições. Os compostos orgânicos foram elaborados com resíduos animais e vegetais, enriquecidos com torta de mamona, MB4® e termofosfato. O enriquecimento mostrou-se eficiente em aumentar a concentração de nutrientes nos compostos. Os compostos orgânicos aumentaram os teores de matéria orgânica do solo (MOS), com destaque para o composto $\mathrm{C}$, que apresentou teores totais mais elevados de $\mathrm{P}, \mathrm{K}, \mathrm{Ca}, \mathrm{Mg}, \mathrm{B}, \mathrm{Cu}, \mathrm{Mn}$ e $\mathrm{Zn}$. A MOS aumentou linearmente com o aumento das doses do composto. A produção e o número de frutos por planta foram maiores com os compostos $\mathrm{B}$ e $\mathrm{C}$, que por sua vez apresentaram maiores concentrações totais de nutrientes. As doses de composto proporcionaram aumento linear na concentração foliar de N. A produção de frutos e o número de frutos por planta apresentaram aumento quadrático com as doses de composto, sem apresentar um ponto de máxima. Termos para Indexação: produção orgânica de manga, rocha moída, análise de solo, nutrição mineral, relação C:N.

\section{ORGANIC COMPOST ON MANGO TREES (Mangifera indica L.) CULTIVATED IN THE SEMI-ARID OF NORTHEAST REGION OF BRAZIL}

\begin{abstract}
The objective of this study was to evaluate the soil chemical characteristics, the nutrient contents in leaves and the production of mango crops grown in the organic system. An experiment was carried out with three composts named A, B and C, using three levels $\left(0,5\right.$ and $\left.10 \mathrm{tha}^{-1}\right)$ of each compost in a commercial production of mango on organic cultivation. The experiment was carried out in a randomized block design, $3 \times 3$ factorial ( 3 composts $\times 3$ levels) and three replications. The organic composts were prepared with animal and vegetable waste, enriched with castor bean, MB4 ${ }^{\circledR}$ and thermo phosphate. The enrichment was efficient in increasing the content of nutrients in composts. The organic composts increased the levels of soil organic matter (SOM), especially in the compost $\mathrm{C}$, whose soil analysis showed higher levels of total $\mathrm{P}, \mathrm{K}, \mathrm{Ca}, \mathrm{Mg}, \mathrm{B}, \mathrm{Cu}, \mathrm{Mn}$ and $\mathrm{Zn}$. The SOM increased linearly with the enhancement of the compost levels. Production and fruit number per plant were higher with the use of the $\mathrm{B}$ and $\mathrm{C}$ composts, which showed higher contents of total nutrients. The compost levels provided a linear increase in $\mathrm{N}$ leaf content. The production of fruit $\left(\mathrm{kg} \mathrm{ha}^{-1}\right)$ and fruit number per plant showed quadratic increase with the compost concentrations, without presenting a maximum point.
\end{abstract}

Index terms: organic production of mango, milled rock, soil analysis, mineral nutrition, C:N rate.

'(Trabalho 080-13). Recebido em: 01-03-2013. Aceito para publicação em: 12-08-2013.Trabalho realizado com recursos da Embrapa e do Banco do Nordeste.

${ }^{2}$ Eng. Agr., Dr. em Solos e Nutrição de Plantas, Pesquisador Embrapa Semiárido, Caixa Postal 23, Petrolina, PE, CEP 56302-970, E-mail: davi.jose@embrapa.br

${ }^{3}$ Eng. Agr., Dr. em Horticultura, Pesquisador Embrapa Semiárido, E-mail: maria.mouco@embrapa.br

${ }^{4}$ Eng. Agr., Dr. em Produção Vegetal, Pesquisador Embrapa Semiárido, E-mail: carlos.gava@embrapa.br

${ }^{5}$ Eng. Agr., Dr. em Ciência do Solo, Pesquisador Embrapa Semiárido, E-mail: vanderlise.giongo@embrapa.br

${ }^{6}$ Eng. Agric., Dr. em Irrigação e Drenagem, Pesquisador Embrapa Semiárido, E-mail: jose-maria.pinto@embrapa.br 


\section{INTRODUÇÃO}

A agricultura orgânica apresenta crescimento contínuo nas diversas partes do mundo. $\mathrm{O}$ total de áreas sob manejo orgânico, incluindo aquelas em fase de conversão, cresceu 3,4 vezes em 11 anos e ocupou uma área de 37 milhões de hectares em 2012. Os continentes de maiores áreas agrícolas sob manejo orgânico são Oceania (12,1 milhões de hectares), Europa (10 milhões de hectares) e América Latina (8,4 milhões de hectares). A produção de manga dobrou em 30 anos, correspondendo a um volume de 35 milhões de toneladas de frutos em 2010, sendo a Ásia, com produção principalmente de mangas nativas, a responsável pela maior produção, seguida pelas Américas e pela África. A manga orgânica tem, atualmente, um mercado crescente e com preços diferenciados, cuja produção mundial de frutos é estimada em 13 milhões de toneladas (MEDINAURRUTIA et al., 2011).

A utilização de resíduos orgânicos, especialmente na forma de composto, que contém altos teores de matéria orgânica, contribui para maior armazenamento de carbono no solo (CLEMENTE et al., 2012), aumento da CTC (MELO et al., 2008; JIMÉNEZ BECKER et al., 2010) e da disponibilidade de nutrientes (BALDI et al., 2010b; JIMÉNEZ BECKER et al., 2010; CLEMENTE et al., 2012), maior atividade e biomassa microbiana (BRAVO et al., 2012; CLEMENTE et al., 2012), maior complexidade dos elementos tóxicos e micronutrientes (CLEMENTE et al., 2012).

Compostos orgânicos produzidos a partir de resíduos animais e vegetais têm sido utilizados pelos agricultores do mundo inteiro por centenas de anos com o objetivo de aumentar a produção agrícola e a qualidade do solo, além de causar menores impactos edáficos, climáticos e econômicos. No entanto, estes produtos apresentam características químicas, físicas e biológicas bastante diversas, como teores de nutrientes e relação $\mathrm{C}: \mathrm{N}$, que conferem uma variabilidade muito alta em termos de qualidade e desempenho agrícola. Dessa forma, a seleção criteriosa do tipo e da fonte dos materiais a serem utilizados, assim como das condições do processo de compostagem são fatores que interferem na qualidade do composto produzido e nos benefícios para o agroecossistema (JIMÉNEZ BECKER et al., 2010).

Este trabalho teve como objetivo avaliar as características químicas do solo, a concentração de nutrientes nas folhas e a produção de mangueiras cultivadas em sistema orgânico, sob irrigação localizada, em função da aplicação de compostos orgânicos, produzidos numa pequena propriedade agrícola do Submédio Vale do São Francisco, região semiárida do Nordeste.

\section{MATERIAL E MÉTODOS}

O trabalho foi realizado na localidade de Santa Helena, zona rural do município de Juazeiro$\mathrm{BA}$, em área de um pequeno produtor, membro da Associação de Produtores Orgânicos da Adutora Caraíba e Adjacências - APROAC. O clima da região é do tipo BSwh', segundo a classificação de Köeppen. O volume médio de chuva é igual a 538,7 mm anuais, com $90 \%$ desse total concentrado nos meses de novembro a abril. A temperatura média varia de $24,3^{\circ} \mathrm{C}$ a $28,5^{\circ} \mathrm{C}$, sendo julho o mês mais frio e novembro o mês mais quente, e a umidade relativa varia de $56 \%$ a $73 \%$, sendo os menores valores obtidos nos meses de setembro a novembro.

$\mathrm{O}$ experimento foi realizado em um Cambissolo, em área de mangueiras (Mangifera indica L.) cultivar Tommy Atkins, com cerca de 15 anos de idade, no espaçamento $10 \times 10 \mathrm{~m}$, em sistema de cultivo convencional. Posteriormente, a propriedade agrícola passou por período de conversão para o sistema de produção orgânica, sendo certificada em 2006 pela Skal International.

Antes da implantação do experimento, o solo da área experimental foi analisado, apresentando, na camada de 0 a $20 \mathrm{~cm}$ de profundidade: matéria orgânica $11,38 \mathrm{~g} \mathrm{~kg}^{-1} ; \mathrm{pH}$ em água 6,9 ; C.E. $0,40 \mathrm{dS}$ $\mathrm{m}^{-1}$; $\mathrm{P}$ disponível $11 \mathrm{mg} \mathrm{dm}^{-3} ; \mathrm{K}$ disponível 0,25 $\mathrm{cmol}_{\mathrm{c}}$

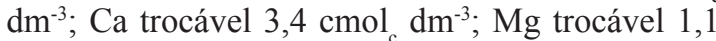
$\mathrm{cmol}_{\mathrm{c}} \mathrm{dm}^{-3} ; \mathrm{Na}$ trocável $0,11 \mathrm{cmol}_{\mathrm{c}} \mathrm{dm}^{-3} ; \mathrm{Al}$ trocável $0,05 \mathrm{cmol} \mathrm{dm}_{\mathrm{c}}^{-3}$; CTC 6,02 $\mathrm{cmol}_{\mathrm{c}} \mathrm{dm}^{-3}$ e V $81 \%$

A irrigação foi realizada diariamente, pelo sistema de microaspersão, com um emissor por planta, sendo a vazão dos emissores de $35 \mathrm{~L} \mathrm{~h}^{-1}$, e as linhas laterais de 36 metros de comprimento, espaçadas de três metros. O coeficiente de uniformidade de distribuição variou entre 69,67 e 90,03\%.

A manutenção da fertilidade do solo, do estado nutricional e fitossanitário das plantas foi realizada conforme procedimento local, por meio da aplicação de resíduos animais e vegetais, produzidos na propriedade ou nas proximidades,pó de rocha, MB4® (fosfática, calcário, gesso), cinzas, caldas (biofertilizantes, repelentes) e outros produtos permitidos pela legislação que regulamenta a produção orgânica no Brasil.

Foram preparados três compostos orgânicos (A, B e C) em 2008 e 2009, com resíduos animais e vegetais gerados na propriedade e nas proximidades da mesma, sendo constituídos por diferentes proporções dos insumos: esterco de caprino, cana- 
de-açúcar (planta inteira), folhas de bananeira, folhas e ramos de mangueira, folhas e ramos de pau-debesouro (Senna spectabilis var. excelsa (Schrad.) H.S. Irwin \& Barneby) e folhas de coqueiro (Tabela 1). Os resíduos vegetais foram seccionados em pedaços de 3 a $5 \mathrm{~cm}$ com uso de uma ensiladeira. Em 2009, os compostos foram preparados com os mesmos insumos, em proporções similares às de 2008, e foram enriquecidos com torta de mamona, MB4 $®$ e termofosfato (Tabela 1).

Nos dois anos, foram montadas duas pilhas de cada composto, nas dimensões $2,0 \mathrm{~m}$ de comprimento x 1,5 m de largura x 1,0 m de altura. As camadas de cada material relacionado na Tabela 1 foram colocadas alternadamente nas pilhas, na sequência apresentada nessa Tabela, na quantidade equivalente à terça parte $(33,3 \mathrm{~cm})$ do total necessário para atingir a altura de $1 \mathrm{~m}$. Durante a fase de compostagem, as pilhas foram irrigadas por dois microaspersores para manutenção da umidade em torno de 75-80 $\%$ e da temperatura entre 36 e $62{ }^{\circ} \mathrm{C}$ adequadas à sobrevivência dos microrganismos.

Os compostos foram revolvidos quinzenalmente, para permitir o arejamento das pilhas e maior eficiência dos organismos no processo de compostagem. Antes do revolvimento foi realizado o monitoramento da temperatura, por meio de um termômetro de solo (Thermometer ${ }^{\circledR}$, modelo TM914-C). O processo foi considerado concluído aos 90 dias após a montagem das pilhas, quando ocorreu a redução da massa a 1/3 do volume inicial e apresentava as características de homogeneidade, plasticidade, partículas de tamanho reduzido, cor escura e brilhante, cheiro de terra mofada e diminuição da umidade para 30-40\%. Os compostos foram analisados para a caracterização dos teores totais de macro e micronutrientes.

Os compostos orgânicos A, B e C (Tabela 1) foram aplicados em doses de $0 ; 5$ e $10 \mathrm{t} \mathrm{ha}^{-1}$, cada um, em área comercial de mangueiras em cultivo orgânico. $\mathrm{O}$ ensaio foi conduzido no delineamento de blocos ao acaso, em esquema fatorial $3 \times 3$ (3 compostos $x 3$ doses), com 3 repetições. A unidade experimental foi constituída por três plantas.

As características avaliadas foram: composição química dos compostos, características químicas do solo dois anos após a implantação do ensaio (final do experimento), teores de nutrientes em folhas coletadas 30 dias antes do florescimento, produção $\mathrm{em} \mathrm{kg} / \mathrm{ha}$, número de frutos por planta e peso médio dos frutos. Os resultados foram submetidos à análise de variância e de regressão, e as médias obtidas para o fator 'compostos' foram comparadas pelo teste de Tukey, a 5\% de probabilidade. As equações de re- gressão foram ajustadas para as variáveis que tiveram efeitos significativos do fator ' doses do composto'.

\section{RESULTADOS E DISCUSSÃO}

\section{Caracterização dos compostos}

A análise da composição química dos compostos mostrou a capacidade dos mesmos em contribuir com a nutrição das plantas (JIMÉNEZ BECKER et al., 2010). O composto A apresentou os maiores teores de $\mathrm{N}$ total, em função do enriquecimento com torta de mamona. No entanto, a relação C:N do composto A foi a mais alta entre os três (Tabela 2), apesar da elevada disponibilidade de nitrogênio proporcionada pela torta de mamona e outros insumos desse composto. As folhas de bananeira que entraram na proporção de $50 \%$ do composto A, contribuíram para manter as maiores concentrações de carbono, pois têm uma rica constituição em celulose $(28,1 \%)$, hemicelulose $(31,7 \%)$ e lignina $(12,4 \%)$ (FIGUEIRÓ; GRACIOLLI, 2011). Além disso, o composto A tem outros constituintes ricos em $\mathrm{C}$ como as folhas de pau-de-besouro e esterco de caprino, em comparação com os demais insumos, resultando em um composto com relação $\mathrm{C}: \mathrm{N}$ mais alta. A planta de cana-de-açúcar, que possui relação $\mathrm{C}: \mathrm{N}$ inicial mais alta $(122,5)$, tem menor concentração de celulose $(24,5 \%)$, hemicelulose (19,9\%) e lignina (4,2\%) (ANDRADE et al., 2004).

De forma geral, a média da relação $\mathrm{C}: \mathrm{N}$ final dos compostos ficou muito próxima de 17:1, na faixa recomendada para uso agrícola, considerada como ideal para a mineralização do nitrogênio. Alguns insumos podem interferir nos teores finais de $\mathrm{C}$ e N e, consequentemente, na relação $\mathrm{C}: \mathrm{N}$ do composto. Gabhane et al. (2012) obtiveram menores teores de carbono orgânico total em compostos enriquecidos com fosfogesso e cinzas, enquanto a adição de açúcar de tâmara e polietilenoglicol proporcionaram aumento dos teores de $\mathrm{N}$ total nos compostos, reduzindo a relação C:N; o açúcar reduziu a relação $\mathrm{C}: \mathrm{N}$ do composto para $11,66: 1$, sendo $8 \%$ menor que o controle.

No composto B, os resíduos vegetais e o enriquecimento com MB4® proporcionaram teores elevados de $\mathrm{Ca}, \mathrm{Mg}$ e $\mathrm{Fe}$, principalmente em comparação com o composto A. Também o composto $\mathrm{C}$ apresentou teores mais elevados de $\mathrm{P}, \mathrm{K}, \mathrm{Ca}, \mathrm{Mg}$, $\mathrm{B}, \mathrm{Cu}, \mathrm{Mn}$ e $\mathrm{Zn}$, refletindo o enriquecimento com termofosfato contendo magnésio e micronutrientes. A adição de torta de mamona e pó de rocha mostrou-se eficiente em aumentar a concentração de nutrientes totais nos compostos.

Os compostos podem atuar como reserva de nutrientes, controlada pelos complexos húmicos, 
permitindo a retenção e reduzindo as perdas de nutrientes no solo (JIMÉNEZ BECKER et al., 2010). Isto é particularmente importante em solos arenosos e de baixa CTC. Contudo, a liberação dos nutrientes no solo em formas disponíveis e a absorção pelas plantas dependem de outros fatores relacionados com características do solo e da planta. Os resultados deste trabalho ratificam a importância da escolha dos insumos e do controle do processo de compostagem para a qualidade do composto produzido (HIGARASHI et al., 2008; JIMÉNEZ BECKER et al., 2010; MONTEMURRO, 2010).

\section{Características químicas do solo}

Comparando as análises de solo antes e depois da conclusão do experimento, verificou-se que os compostos orgânicos influenciaram algumas características químicas do solo (Tabela 3 ).

Os compostos orgânicos proporcionaram aumento dos teores de matéria orgânica do solo (MOS), em relação aos teores existentes no solo antes do início do experimento. O composto C, embora não tenha apresentado a maior relação $\mathrm{C}: \mathrm{N}$, conferiu aumento significativo da MOS em comparação com os outros compostos (Tabela 3). O composto $\mathrm{C}$ mostrou a composição mais equilibrada, contendo cana-de-açúcar, folha de bananeira, folha de coqueiro, folhas de mangueira e esterco de caprino. $\mathrm{O}$ efeito de compostos orgânicos sobre a MOS foi também encontrado em outros trabalhos. Em um ensaio conduzido durante nove anos em um cultivo de nectarineiras, foram observados efeitos positivos do tratamento com um composto de resíduos sólidos urbanos sobre a MOS (TOSELLI, 2010), havendo ainda aumento do $\mathrm{C}$ da biomassa microbiana, dos teores de N, P e K no solo (BALDI et al., 2010b) e aumento da produção e do tempo de vida de novas raízes (BALDI et al., 2010a). Clemente et al. (2012) também obtiveram maiores teores de MOS, dos nutrientes $\mathrm{P}, \mathrm{K}$ e $\mathrm{Mn}$, e maior atividade microbiana com a aplicação de um composto semelhante.

$\mathrm{O}$ pH do solo e a condutividade elétrica também foram alterados pelos compostos, mas em pequena intensidade e apenas na profundidade de 20 a $40 \mathrm{~cm}$. Ramos et al. (2009) obtiveram aumentos de $\mathrm{pH}$ e MOS em resposta a um resíduo de algodão compostado na cultura do feijoeiro. As demais características do solo não foram alteradas pelos diferentes compostos no presente trabalho.

A MOS aumentou com o aumento das doses do composto. Houve efeito linear das doses dos compostos sobre a MOS (Tabela 5). Rocha et al. (2004) obtiveram aumento da MOS, P e Ca no solo devido à aplicação de doses crescentes de um biossólido em um povoamento de Eucalyptus grandis. Em cultivos de alface, também foram obtidos aumentos da MOS em resposta a doses de diferentes compostos (SANTOS et al., 1999; YURI et al., 2004; MARCHI et al., 2008). Em um cultivo de nectarineiras, Toselli (2010) obteve resultados semelhantes para as doses de 5 e $10 \mathrm{tha}^{-1}$ do composto com o aumento do teor de MOS de 16 para $30 \mathrm{~g} \mathrm{~kg}^{-1}$.

Concentração de nutrientes nas folhas e características de produção da mangueira

As concentrações foliares de N, P, K e Ca não diferiram significativamente entre os compostos avaliados (Tabela 4). Souza et al. (2005) obtiveram aumentos dos teores de $\mathrm{P}, \mathrm{K}$ e $\mathrm{Mg}$ em folhas de alface em função da aplicação de um composto elaborado com resíduos vegetais e esterco bovino. $\mathrm{O}$ efeito da qualidade do composto sobre os teores de $\mathrm{N}$ e a produção de alface também foi reportado por Montemurro (2010).

Embora não tenha ocorrido diferença entre os compostos para a concentração foliar de $\mathrm{N}$, as doses dos compostos proporcionaram aumento linear na concentração foliar de $\mathrm{N}$ (Tabela 5). A aplicação de doses do composto comercial Organosuper ${ }^{\circledR}$ de 0 ; 15; 30; 60 e $120 \mathrm{t} \mathrm{ha}^{-1}$ proporcionou maior acúmulo de $\mathrm{N}$ e $\mathrm{P}$ em mudas de pinhão-manso (SCHIAVO et al., 2010).

A produção e o número de frutos por planta foram maiores com o uso do composto $\mathrm{B}$, embora não tenha diferido do composto $\mathrm{C}$, porém ambos mostraram maiores teores totais de nutrientes (Tabela 4). As doses de composto proporcionaram um comportamento quadrático aos dados de produção e número de frutos, sem apresentar, contudo, um ponto de máxima. Os rendimentos em produção de nectarinas 'Stark Red Gold' aumentaram de $13,5 \mathrm{t} \mathrm{ha}^{-1}$ para 19,9 $\mathrm{t} \mathrm{ha}^{-1}$ em função do aumento de doses de composto orgânico, de MOS e da maior disponibilidade de nutrientes N, P e K no solo (BALDI et al., 2010b; TOSELLI, 2010.) Por outro lado, Bravo et al. (2012) relatam que a aplicação de composto constituído por material de poda de árvores urbanas e resíduos orgânicos domésticos aumentou a fixação de ${ }^{13} \mathrm{CO}_{2}$, promovendo o crescimento da parte aérea e das raízes de nectarineiras, mas não afetou a biomassa dos frutos nem a absorção de nitrogênio. 
TABELA 1 - Composição, teores de carbono e nitrogênio, relação C:N e proporção dos insumos usados na elaboração dos compostos na APROAC. Juazeiro-BA, 2009.

\begin{tabular}{lcccccc}
\hline Insumo & $\mathrm{C}$ & $\mathrm{N}$ & $\mathrm{C}: \mathrm{N}$ & Composto A & Composto B & Composto C \\
\hline & $---\mathrm{g} \mathrm{kg}^{-1}$--- & & ---- \% ---- & ---- \% ---- & $-----\%$----- \\
Planta de Cana-de-açúcar & 497,6 & 4,06 & 122,5 & 0 & 50 & 23,3 \\
Folha de Bananeira & 465,1 & 8,12 & 57,3 & 50 & 0 & 23,3 \\
Folha de Coqueiro & 517,5 & 8,12 & 63,7 & 0 & 0 & 23,4 \\
Folha de Mangueira & 506,9 & 11,0 & 46,0 & 0 & 12 & 7 \\
Folha de Pau-de-Besouro & 508,5 & 17,1 & 29,2 & 20 & 10 & 0 \\
Esterco de Caprino & 174,7 & 12,2 & 14,3 & 20 & 25 & 20 \\
Torta de Mamona & 350,0 & 60,0 & 5,8 & 10 & 0 & 0 \\
MB4® & & & & 0 & 3 & 0 \\
Termofosfato & & & & 0 & 0 & 3 \\
\hline
\end{tabular}

TABELA 2 - Relação C:N e composição química dos compostos elaborados na APROAC e utilizados no experimento. Juazeiro-BA, 2010.

\begin{tabular}{|c|c|c|c|c|c|c|c|c|c|c|c|c|c|}
\hline Com & $: N$ & C & $\mathrm{N}$ & $\mathrm{P}$ & K & $\mathrm{Ca}$ & $\mathrm{Mg}$ & S & B & $\mathrm{Cu}$ & $\mathrm{Fe}$ & $\mathrm{Mn}$ & $\mathrm{Zn}$ \\
\hline & & \multicolumn{12}{|c|}{ 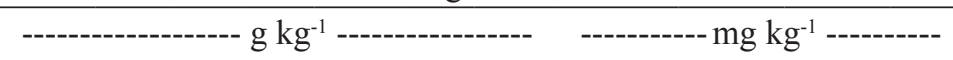 } \\
\hline A & 19,6 & 4,7 & 164 & 3,7 & 2,8 & 9,4 & 3,6 & 1,99 & 42 & 15 & 5584 & 302 & 87 \\
\hline B & 13 , & 6 & 12,2 & 2,5 & 2,8 & 15,1 & 11,9 & 1,77 & 41 & 13 & 11015 & 360 & 69 \\
\hline $\mathrm{C}$ & 13,5 & 141,2 & 10,4 & 10,5 & 3,6 & 22,8 & 8,2 & 1,37 & 99 & 23 & 7372 & 1099 & 416 \\
\hline
\end{tabular}

TABELA 3 - Teores de matéria orgânica do solo (MOS), pH, condutividade elétrica (CE), capacidade de troca de cátions (CTC) e percentagem de saturação por bases (V) em amostras de solo coletadas nas camadas de 0-20 cm e 20-40 cm de profundidade, em função da adubação com diferentes compostos orgânicos. Juazeiro-BA, 2010.

\begin{tabular}{cccccc}
\hline Composto & MOS & $\mathrm{pH}$ & $\mathrm{CE}$ & $\mathrm{CTC}$ & $\mathrm{V}$ \\
\hline & $\mathrm{g} \mathrm{kg}^{-1}$ & \multicolumn{5}{c}{$0 \mathrm{dS} \mathrm{m}^{-1}$} & $\mathrm{cmolc} \mathrm{dm}^{-3}$ & $\%$ \\
\hline \multicolumn{7}{c}{} \\
& & & $0 \mathrm{~cm}$ & \\
$\mathrm{~A}$ & $14,20 \mathrm{~b}$ & $7,31 \mathrm{a}$ & $0,52 \mathrm{a}$ & $14,36 \mathrm{a}$ & $98 \mathrm{a}$ \\
$\mathrm{B}$ & $17,49 \mathrm{~b}$ & $7,20 \mathrm{a}$ & $0,54 \mathrm{a}$ & $15,27 \mathrm{a}$ & $98 \mathrm{a}$ \\
$\mathrm{C}$ & $23,46 \mathrm{a}$ & $7,31 \mathrm{a}$ & $0,53 \mathrm{a}$ & $14,74 \mathrm{a}$ & $98 \mathrm{a}$ \\
& & & $20-40 \mathrm{~cm}$ & & \\
\hline $\mathrm{A}$ & $7,39 \mathrm{a}$ & $7,30 \mathrm{a}$ & $0,35 \mathrm{ab}$ & $12,95 \mathrm{a}$ & $96 \mathrm{a}$ \\
\hline $\mathrm{B}$ & $7,79 \mathrm{a}$ & $7,19 \mathrm{~b}$ & $0,41 \mathrm{a}$ & $13,48 \mathrm{a}$ & $97 \mathrm{a}$ \\
$\mathrm{C}$ & $7,43 \mathrm{a}$ & $7,14 \mathrm{~b}$ & $0,26 \mathrm{~b}$ & $12,83 \mathrm{a}$ & $96 \mathrm{a}$ \\
\hline
\end{tabular}

Médias seguidas pela mesma letra nas colunas não diferem entre si, pelo teste de Tukey $(p<0,05)$ 
TABELA 4 - Teores de nitrogênio $(\mathrm{N})$, fósforo $(\mathrm{P})$, potássio $(\mathrm{K})$ e cálcio $(\mathrm{Ca})$ em folhas de mangueira coletadas 30 dias antes do florescimento, e valores de produção (PROD), número de frutos por planta (NFRU) e peso médio de frutos (PMF) em função da adubação com diferentes compostos orgânicos. Juazeiro-BA, 2010

\begin{tabular}{cccccccc}
\hline Composto & $\mathrm{N}$ & $\mathrm{P}$ & $\mathrm{K}$ & $\mathrm{Ca}$ & PROD & NFRU & PMF \\
\hline & ---------------- & $\mathrm{g} \mathrm{kg}^{-1}$ & ------------- & $---\mathrm{kg} \mathrm{ha}^{-1}---$ & & $--\mathrm{g} \mathrm{---}$ \\
$\mathrm{A}$ & $16,73 \mathrm{a}$ & $1,04 \mathrm{a}$ & $10,89 \mathrm{a}$ & $19,24 \mathrm{a}$ & $17771,02 \mathrm{~b}$ & $343,7 \mathrm{~b}$ & $0,518 \mathrm{a}$ \\
$\mathrm{B}$ & $16,63 \mathrm{a}$ & $1,07 \mathrm{a}$ & $11,62 \mathrm{a}$ & $17,89 \mathrm{a}$ & $23726,85 \mathrm{a}$ & $449,1 \mathrm{a}$ & $0,529 \mathrm{a}$ \\
$\mathrm{C}$ & $15,66 \mathrm{a}$ & $0,94 \mathrm{a}$ & $11,28 \mathrm{a}$ & $18,86 \mathrm{a}$ & $23265,56 \mathrm{ab}$ & $447,1 \mathrm{ab}$ & $0,521 \mathrm{a}$ \\
\hline
\end{tabular}

Médias seguidas pela mesma letra nas colunas não diferem entre si, pelo teste de Tukey $(\mathrm{p}<0,05)$

TABELA 5 - Equações de regressão para teores de matéria orgânica no solo (MOS) em amostras de solo coletadas de 0-20 cm de profundidade e concentração foliar de nitrogênio $(\mathrm{N})$, produção de frutos (PROD) e número de frutos por planta (NFRU) em mangueiras submetidas a três doses de compostos orgânicos. Juazeiro-BA, 2010.

\begin{tabular}{clc}
\hline Variável & \multicolumn{1}{c}{ Equação } & $\mathrm{R}^{2}$ \\
\hline MOS & $\hat{\mathrm{y}}=12,2+1,24 * * * \mathrm{x}$ & 0,850 \\
$\mathrm{~N}$ & $\hat{\mathrm{y}}=14,76+0,316^{*} \mathrm{x}$ & 0,991 \\
PROD & $\hat{\mathrm{y}}=22678,15-2018,63 * \mathrm{x}+216^{* *} \mathrm{x}^{2}$ & 0,999 \\
NFRU & $\hat{\mathrm{y}}=431,05-38,23 * \mathrm{x}+4,162 * * \mathrm{x}^{2}$ & 0,999 \\
\hline
\end{tabular}

\section{CONCLUSÃO}

1- A adição de torta de mamona, MB4® ou termofosfato mostra-se eficiente em aumentar o teor de nutrientes nos compostos.

2-Os compostos orgânicos proporcionam aumento dos teores de matéria orgânica do solo, com destaque para o composto $\mathrm{C}$, enriquecido com termofosfato, que apresenta teores totais mais elevados de $\mathrm{P}, \mathrm{K}, \mathrm{Ca}, \mathrm{Mg}, \mathrm{B}, \mathrm{Cu}, \mathrm{Mn}$ e $\mathrm{Zn}$.

3- A concentração de $\mathrm{N}$ nas folhas da mangueira aumenta linearmente com as doses de composto.

4- A produção e o número de frutos por planta são maiores com os compostos $\mathrm{B}$ e $\mathrm{C}$, enriquecidos com MB4 ${ }^{\circledR}$ e termofosfato, respectivamente, que apresentam maiores concentrações totais de nutrientes.

\section{AGRADECIMENTOS}

Ao Banco do Nordeste, pelo suporte financeiro; ao produtor rural Francisco Morais Alves e sua família, pela disponibilização da área e colaboração nos trabalhos de campo; ao assistente de pesquisa Francisco Costa de Aquino, bolsistas e estagiários da Embrapa Semiárido, pela colaboração na execução desta pesquisa.

\section{REFERÊNCIAS}

ANDRADE, J.B.; FERRARI JUNIOR, E.; POSSENTI, R.A.; OTSUK, I.P.; ZIMBACK, L.; LANDELL, M.G.A. Composição química de genótipos de cana-de-açúcar em duas idades, para fins de nutrição animal. Bragantia, Campinas, v.63, n.3, p.341-349, 2004.

BALDI, E., TOSELLI, M., EISSENSTAT, D.M., MARANGONI, B. Organic fertilization leads to increased peach root production and lifespan. Tree Physiology, Oxford, v. 30, n.11, p. 1373-1382, 2010a. 
BALDI, E.; TOSELLI, M.; MARCOLINI, G.; QUARTIERI, M.; CIRILLO, E.; INNOCENTI, A.; MARANGONI, B. Compost can successfully replace mineral fertilizers in the nutrient management of commercial peach orchard. Soil Use and Management, Oxford, v. 26, n. 3, p. 346-353, 2010 b.

BRAVO, K.; TOSELLI, M.; BALDI, E.; MARCOLINI, G.; SORRENTI, G; QUARTIERI, M.; MARANGONI, B. Effect of organic fertilization on carbon assimilation and partitioning in bearing nectarine trees. Scientia Horticulturae, Amsterdam, v. 137, p. 100-106, 2012.

CLEMENTE R.; WALKER, D.J.; PARDO, T.; MARTÍNEZ-FERNÁNDEZ, D.; BERNAL, M.P. The use of a halophytic plant species and organic amendments for the remediation of a trace elementscontaminated soil under semi-arid conditions. Journal of Hazardous Materials, Amsterdam, v. 223-224, p. 63-71, 2012.

FIGUEIRÓ, G.G.; GRACIOLLI, L.A. Influência da composição química do substrato no cultivo de Pleurotus florida. Ciência e Agrotecnologia, Lavras, v. 35, n.5, p.924-930, 2011.

GABHANE, J.; PRINCE WILLIAM, SPM.; BIDYADHAR , R.; BHILAWE, P.; ANAND, D.; VAIDYA, A.N.; WATE, S.R. Additives aided composting of green waste: effects on organic matter degradation, compost maturity, and quality of the finished compost. Bioresource Technology, Essex, v.114, p.382-388, 2012.

HIGARASHI, M.M.; COLDEBELla, A.; OLIVEIRA, P.A.V.; KUNZ, A. MATTEI, R.; SILVA, V.S.; AMARAL, A.L. Concentração de macronutrientes e metais pesados em maravalha de unidade de suínos em cama sobreposta. Revista Brasileira de Engenharia Agrícola e Ambiental, Campina Grande, v.12, p.311-317, 2008.

JIMÉNEZ BECKER, S.; EBRAHIMZADEH, A.; PLAZA HERRADA, B. M.; AND LAO, M. T. Characterization of compost based on crop residues: changes in some chemical and physical properties of the soil after applying the compost as organic amendment. Communications in Soil Science and Plant Analysis, New York, v. 41, n. 5-8, p. 696-708, 2010.
MARCHI, E.C.S.; ALVARENGA, M.A.R.; MARCHI, G; SILVA, C..A.; SOUZA FILHO, J.L. Efeito da adubação orgânica sobre as frações de carbono de solos cultivados com alface americana. Ciência e Agrotecnologia, Lavras, v. 32, n. 6, p. 1760-1766, 2008.

MEDINA-URRUTIA, V.M.; VÁZQUEZ-GARCÍA, M.; VIRGEN-CALLEROS, G. Organic mango production in Mexico: status of orchard management. Acta Horticulturae, The Hague, v. 894, p. 255-263, 2011.

MELO, L.C.A.; SILVA, C.A.; DIAS, B.O. Caracterização da matriz orgânica de resíduos de origens diversificadas. Revista Brasileira de Ciência do Solo, Viçosa, MG, v.32, n.1, p.101-110, 2008.

MONTEMURRO, F. Are organic N fertilizing strategies able to improve lettuce yield, use of nitrogen and $\mathrm{N}$ status? Journal of Plant Nutrition, New York,v. 33, n.13, p.1980-1997, 2010.

RAMOS, S.J.; ALVES, D.S.; FERNANDES, L.A.; COSTA, C.A. Rendimento de feijão e alterações no pH e na matéria orgânica do solo em função de doses de composto de resíduo de algodão. Ciência Rural, Santa Maria, v.39, p.1572-1576, 2009.

ROCHA, G.N.; GONÇALVES J.L.M.; MOURA, I.M. Mudanças da fertilidade do solo e crescimento de um povoamento de Eucalyptus grandis fertilizado com biossólido. Revista Brasileira de Ciência do Solo, Viçosa, MG, v. 28, p. 623-639, 2004.

SANTOS, I.C.; CASALI, V.W.D.; MIRANDA, G.V. Teores de metais pesados, $\mathrm{K}$ e Na, no substrato, em função de doses de composto orgânico de lixo urbano e de cultivares de alface. Ciência Rural, Santa Maria, v. 29, n. 3, p. 415-421, 1999.

SCHIAVO, J.A.; SILVA, C.A.; ROSSET, J.S. SECRETTI, M.L.; SOUSA, R.A.C.; CAPPI, N. Composto orgânico e inoculação micorrízica na produção de mudas de pinhão manso. Pesquisa Agropecuária Tropical, Goiânia, v. 40, n. 3, p. 322-329, 2010. 
SOUZA, P.A.; NEGREIROS, M.Z.; MENEZES, J.B.; BEZERRA NETO, F.; SOUZA, G.L.F.M.; CARNEIRO, C.R; QUEIROGA，R.C.F. Características químicas de alface cultivada sob efeito residual da adubação com composto orgânico. Horticultura Brasileira, Brasília, v. 23, n.3, p. 754757, 2005.

TOSELLI, M. Nutritional implications of organic management in fruit tree production. Acta Horticulturae, The Hague, v. 868, p. 41-48, 2010.
YURI, J.E.; RESENDE, G.M.; RODRIGUES JÚNIOR, J.C.; MOTA, J.H.; SOUZA, R.J. Efeito de composto orgânico sobre a produção e características comerciais de alface americana. Horticultura Brasileira, Brasília, v.22, n.1, p. 127-130, 2004. 